\title{
Inveterate Posterior Shoulder Dislocation Treated by Sub-Scapular Transfer, about One Case
}

\section{Koné Samba ${ }^{1 *}$, Dogba Eric ${ }^{1}$, Kouassi Adélaide², Ledion Anicet ${ }^{1}$, Adibo Godefroi ${ }^{1}$, Ote Pacome $^{1}$, Be Jeancis ${ }^{1}$, Bana Abdoulaye1, Touré Stanislas ${ }^{1}$, Agoh Serge ${ }^{1}$}

\author{
${ }^{1}$ Service de Traumatologie Orthopédie CHU de Cocody, Abidjan, Cote d'Ivoire \\ ${ }^{2}$ Service de Traumatologie Orthopédie CHU de Bouaké, Bouaké, Cote d'Ivoire \\ Email: *ksamba06@gmail.com
}

How to cite this paper: Samba, K., Eric, D., Adélaide, K., Anicet, L., Godefroi, A., Pacome, O., Jeancis, B., Abdoulaye, B., Stanislas, T. and Serge, A. (2017) Inveterate Posterior Shoulder Dislocation Treated by Sub-Scapular Transfer, about One Case. Open Journal of Orthopedics, 7, 301-307. https://doi.org/10.4236/ojo.2017.710030

Received: July 27, 2017

Accepted: October 10, 2017

Published: October 13, 2017

Copyright $\odot 2017$ by authors and Scientific Research Publishing Inc. This work is licensed under the Creative Commons Attribution International License (CC BY 4.0).

http://creativecommons.org/licenses/by/4.0/

\begin{abstract}
Glena-humeral dislocation is rare. More than the half of those dislocations is unremarked because the diagnostic is not an emergency. A delayed diagnostic alters the functional prognostic and sometimes forces to do a complex surgery with complications whose success rate remains uncertain. We report a case of inveterate posterior shoulder dislocation on a 42-year-old adult occurred during a sport accident and treated by sub-scapulars transfer (modified Mac Laughlin's technical). The aim of this article is to highlight this pathology to ameliorate its diagnostic rate indeed prognostic. Our diagnostic approach and injury, noticed during surgery and therapeutic propositions, are described at the light of literature data on this subject.
\end{abstract}

\section{Keywords}

Inveterate Posterior Shoulder Dislocation

\section{Introduction}

Inveterate posterior shoulder dislocation (IPSD) is extremely rare [1]. They always pose nosological, diagnostic and therapeutic problems.

The clinical and radiological symptomatology is well elucidated, but the diagnosis in emergency is sometimes unnoticed; because clinical signs are subtle and less expressive and radiologic imaging sometimes inadequate or difficult to interpret. It is all the interest to have correct standard radiographies which have to conduct at less doubt to a scanner prescription or a magnetic resonance imaging (MRI). 
The diagnostic delay alters the functional prognostic. A posterior shoulder dislocation (PSD), non-reduced after three weeks, is said to be inveterate [2].

The treatment for an inveterate dislocation is most of the time surgical [1] [3]. It uses osseous (blockading, osteotomy, arthroplasty) or sub-scapular-muscular (transfer or tenodesis) gestures. We report an IPSD observation, on a leisure athlete treated by McLaughlin's technical which we have modified.

\section{Case Report}

It's about a 42-year-old man, who had a left shoulder traumatism during basketball match. This leisure athlete presented a pain on the left shoulder, and functional impotence with a limitation of the external rotation. His clinical aspect has been linked to a contusion without radiological investigations. Analgesic and anti-inflammatory drugs were prescribed.

In front of continuing (pain, major functional impotence of the limb, fixed internal) of clinical signs, he decided to consult in specialized environment.

At three weeks, post-traumatism clinical examination put evidence on a local pain, a shoulder blocked in adduction and fixed internal rotation, with absence of vascular-nervous trouble. The shoulder mobility was:

Abduction: $45^{\circ}$ /Adduction fixed; Antepulsion: $70^{\circ} /$ Retropulsion was impossible; Internal rotation: $80^{\circ} /$ External rotation: $30^{\circ}$.

Shoulder radiography (Figure 1) showed a posterior shoulder dislocation. A complementary MRI confirms the existence of a humeral cephalic anterior nick (Mac Laughlin's nick) and shows its expansion (Figure 2). The retained diagnostic is inveterate posterior gleno-humeral dislocation, with cephalic nick less than $50 \%$.

We realized a bloody reduction by delto-pectoral anterior way. The filling of the nick is done by a pedicle transfer of the sub-scapular tendon (Mac Laughlin's technical) protected by a screw supported by washer (Figure 3); supra-supinatus suture. The clinical examination per surgery showed a stable shoulder with a normal mobility sector (Table 1). During post surgery time, shoulder has been mobilised inside a splint with abduction cushion, in neutral rotation for 4 weeks, associated to re-education. The resumption of its socio-professional activities
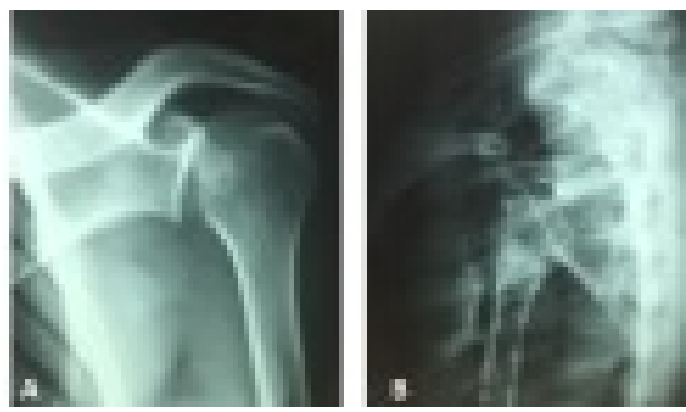

Figure 1. Shoulder radiography disappearance of the glenohumeral joint with the double contour appearance. (A) Antero-posterior radiograph; (B) Medio-lateral radiograph. 


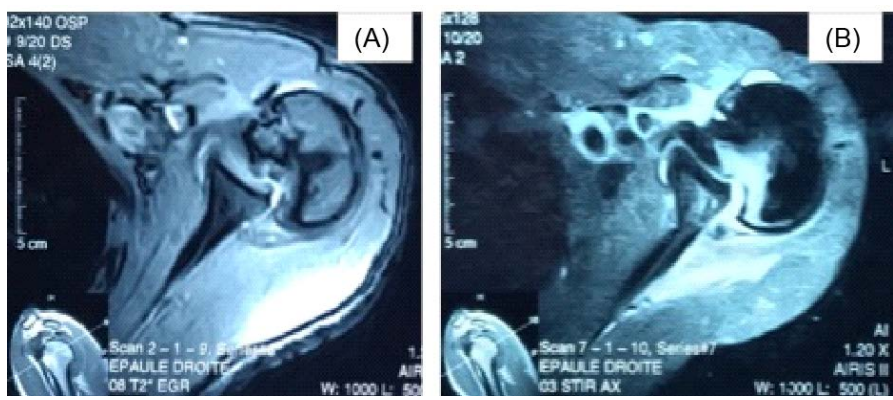

Figure 2. MRI Shoulder showing humeral cephalic anterior nick (Mac Laughlin's nick). (A) Axial incidence; (B) Axial incidence at STIR.
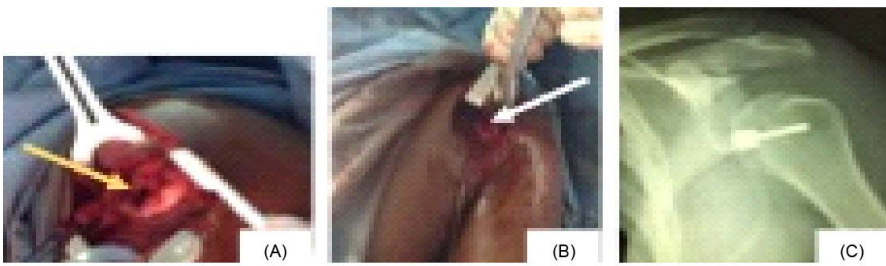

Figure 3. Per-operative view (A \& B). (A) humeral cephalic nick (yellow arrow); (B) screwing on washer (white arrow); (C) post-operative shoulder radiography.

Table 1. Comparative shoulder mobility.

\begin{tabular}{ccc}
\hline Shoulder mobility & Pré-opérative & Post-opérative \\
\hline Abduction & $30^{\circ}$ & $120^{\circ}$ \\
Adduction & fixed & $40^{\circ}$ \\
Antepulsion & $70^{\circ}$ & $160^{\circ}$ \\
Retropulsion & impossible & $40^{\circ}$ \\
Internal rotation & $50^{\circ}$ & $80^{\circ}$ \\
External rotation & Impossible & $35^{\circ}$
\end{tabular}

was possible from the $5^{\text {th }}$ month. After 18 months, a clinical evaluation by Constant's [4] score was 70\% (Pain 10/15: activity level; 18/20: Mobility; 22/40: Force 20/25).

At the radiographic plan there was no omarthrosis sign, nor calcification after 18 months of follow-up.

\section{Discussion}

The postponement of this unusual clinical case that occurred in a young adult was aimed at highlighting this particular entity through an analysis of our diagnosis and discussing the therapeutic modalities. Posterior dislocation of the shoulder is a rare diagnosis [3] [5]. The posterior luxation of the shoulder represents $2 \%$ of the glenohumeral dislocations [6]. These dislocations occur most often during convulsive seizures (epilepsy, ethylism, electroshock). The 
dislocation occurs during flexion, adduction and internal rotation movement. In some cases, a direct anteroposterior shock caused dislocation as was the case for our patient (sports accident). Our clinical case is distinguished by its posterior topography (uncommon variety) and inverted character (unusual form).

The lack of initial diagnosis is in agreement with the conclusions of some authors who consider that the posterior dislocations are difficult of diagnostic and unnoticed in $60 \%$ of the cases [7] [8]. This misunderstanding will evolve into the inveterate form when it exceeds 3 weeks.

The posterior dislocation of the shoulder, constitutes a diagnostic and therapeutic challenge as was in inverted form.

Clinically, the main signs to be sought are: protrusion of the coracoid process, adduction of the arm in internal rotation fixed, the anterior relief of the deltoid appears hollowed out (Figure 4).

On the standard X-rays, the front of the shoulder should look for the appearance in double contour and the sign of the bulb at the humeral head. These signs are very discreet and they can go unnoticed by a quick reading of $\mathrm{X}$-rays. It is all the interest to have a frontal incidence well perpendicular to the plane of the scapula and not to the frontal plane of the patient to avoid the pitfalls diagnosis.

If for some [3] [9] specific additional impacts, the diagnostic doubt is overcome, our preference is for nuclear imaging.

This has a diagnostic, therapeutic and prognostic value. It allows to better visualize the lesion and to look for possible associated lesions [10] (the importance of the Mac Laughlin notch, musculo-tendon or bone lesion). In addition, our patient had a supra-supinatus head injury with an involvement of the anterior edge of the glenoid lesion (Bankart's lesion) Figure 5.

The functional prognosis depends on early diagnosis and timing of treatment.

The particularity of the treatment of inveterate posterior dislocations compared to recent dislocations is mainly the difficulty of secondary reduction (linked to the retraction of the soft parts) and the choice of surgical technique according to the size of the cephalic notch. Randelli defines 04 stages of cephalic notch of Mac Laughlin [11].
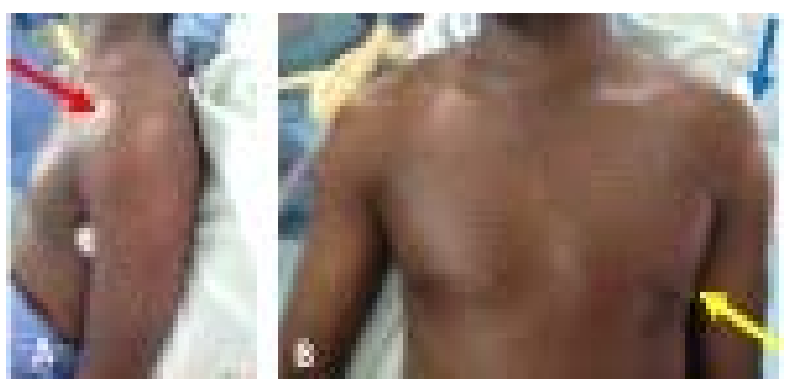

Figure 4. Preoperative clinical aspect. (A) projection of the coracoide (red arrow); (B) front view, fall of the stump (blue arrow) Adduction of the arm (yellow arrow). 


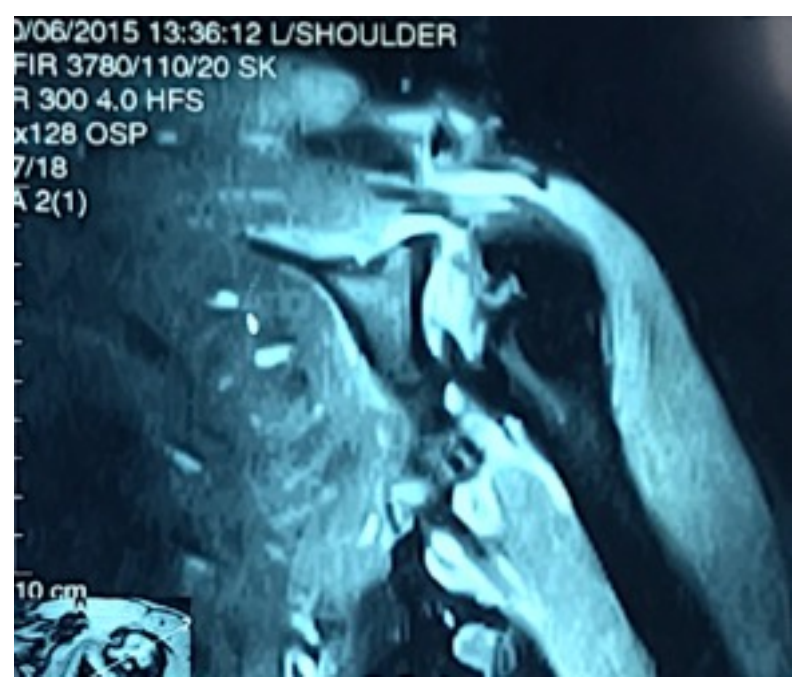

Figure 5. MRI Shoulder showing supra-supinatus's lesion/coronal STIR.

Behind an inveterate posterior dislocations form wicth delay of less than 6 weeks and with a Randelli stage 1 cephalic notch, some authors [2] [3] propose that no-bloody reduction should be always attempted.

After 6 weeks of delay, and regardless of the size of the cephalic notch, the literature proposes a surgical reduction associated with various notch filling techniques (graft, arthroplasty, osteotomy, subscapular transfer or tenodesis, arthrodesis).

In our patient we performed a bloody reduction and transfer of the subscapularis for the following reasons: the existence of lesions associated, the size of the notch (stage 1) didn't required osseous graft.

This clinical case reveals a third parameter to be taken account in addition to diagnostic delay and characters of the cephalic notch: it's the associated lesions.

According to the literature it would seem that the filling of the notch by the transfer of the tendon of the sub-scapular is more effective than at the tenodesis of the latter [2] [5].

Mac Laughlin [12] was the first to describe a filling technique by simply transferring sub-scapular tendon into the cephalic notch with osteo-suture. Neer and Hawkins [13], on the other hand, used bone filling by transferring the trochin. For them [13] using bone-to-bone interface avoids the swallowing of the notch by opposing a bony surface to the rim of the glenoid [5] [7]. For our patient we modified Mac Laughlin [9] technique by using an screwing. For us the use of a screw resting on a washer stabilizes the assembly more efficiently than the osteo-suture described by Mac Laughlin [12].

At the functional plan constant's score was accetable for our patient and it is satisfied to have obtained some mobility (constant's score was 70\%) despite a slight abduction and anté-pulsion deficit.

Whatever the chosen indication, the diagnostic delay alters the functional prognosis as it was our patient case. An early diagnostic is the key of a favour- 
able prognosis.

Some authors [14] believe that closed reductions provide better functional results than bloody reductions. The diagnostic delay and surgical management time are also major prognostic factors.

\section{Conclusions}

Posterior shoulder dislocations are rare lesions [1]. The diagnostic is sometimes unknown in emergency thus constituting a real challenge. An attentive anamnesis, and clinical examination associated to quality radiologic investigations will permit to reduce the delayed diagnostic number.

The ISPD mostly obliges to a complex surgery with complications whose success rate remains uncertain.

The best treatment stays an early diagnostic of these posterior dislocations at acute stage.

\section{Consent}

The patient and the families were informed that data from the case would be submitted for publication and provided their consent accordingly.

\section{Conflict of Interests}

The authors declare that they have no competing interests.

\section{Author's Contributions}

All the authors contributed of the writing of this manuscript and have read and approved the final version.

\section{References}

[1] Augereau, B., Leyder, P. and Et Apoil, A. (1983) Treatment of Inveterate Posterior Shoulder Dislocation by Double Approach and Retro-Glenoid Bone Abutment. Revue de Chirurgie Orthopedique, 69, 289-290.

[2] Khiami, F. and Rolland, E. (2006) Surgical Treatment of Inveterate Posterior Shoulder Dislocation. Journal de Traumatologie du Sport, 23, 164-169.

[3] Cunningham, G. and Hoffmeyer, P. (2011) Development, Posterior Shoulder Dislocation, Diagnostic and Therapeutic Challenges. Revue Médicale Suisse, 7, 2489-2493.

[4] Christopher, R., et al. (2008) A Review of the Constant Score: Modifications and Guidelines for Its use. Journal of Shoulder and Elbow Surgery, 17, 355-361. https://doi.org/10.1016/j.jse.2007.06.022

[5] El Aaoui, A., Rabhi, I., Bah, A., et al. (2015) Posterior Dislocation of the Shoulder in an Athlete Treated by the Sub-Scapular Pedicle Transfer. Pan African Medical Journal, 22, 353.

[6] Cave, E.F., Burke, J.F. and Boyd, R.J. (1974) Trauma Management. Year Book Medical Publishers, Incorporated.

[7] Rowe, C.R. and Zarins, B. (1982) Chronic Unreduced Dislocations of the Shoulder. The Journal of Bone and Joint Surgery, American Volume, 64, 494-505. https://doi.org/10.2106/00004623-198264040-00004 
[8] Mestdagh, H., et al. (1994) Traumatic Posterior Dislocation of the Shoulder in Adults. Apropos of 25 Cases. Annales de Chirurgie, 48, 355-363.

[9] Robinson, C.M., Seah, M. and Akhtar, M.A. (2011) The Epidemiology, Risk of Recurrence, and Functional Outcome after an Acute Traumatic Posterior Dislocation of the Shoulder. The Journal of Bone and Joint Surgery, American Volume, 93, 1605-1613. https://doi.org/10.2106/JBJS.J.00973

[10] Aldebeyan, S., Aoude, A. and Van Lancker, H. (2015) Traumatic Posterior Shoulder Dislocation with a Large Engaging Hill-Sachs Lesion: A Case Report and a Novel Splinting Technique. American Journal of Emergency Medicine, 26.

[11] Randelli, M. and Gambrioli, P.L. (1986) Glenohumeral Osteometry by Computed Tomography in Normal and Unstable Shoulders. Clinical Orthopaedics, 208, 151-156.

[12] Mac Laughlin, H.L. (1952) Posterior Dislocation of the Shoulder. The Journal of Bone and Joint Surgery, American Volume, 34-A, 584-590. https://doi.org/10.2106/00004623-195234030-00011

[13] Hawkins, R.J., Neer, C.S., Pianta, R.M. and Mendoza, F.X. (1987) Locked Posterior Dislocation of the Shoulder. The Journal of Bone and Joint Surgery, American Volume, 69, 9-18. https://doi.org/10.2106/00004623-198769010-00003

[14] Schliemann, B., Muder, D., Gessmann, J., et al. (2011) Locked Posterior Shoulder Dislocation: Treatment Options and Clinical Outcomes. Archives of Orthopaedic and Trauma Surgery, 131, 1727-1734. 